\title{
Analogue Experimental Study of Fiber Formation on Two-Wheel Spinner
}

\author{
Benjamin Bizjan* - Brane Širok - Marko Blagojevič \\ University of Ljubljana, Faculty of Mechanical Engineering, Slovenia
}

In this paper, the process of mineral fiber formation was investigated experimentally on a two-wheel spinner by means of high-speed imaging. Analogue isomalt melt was fiberized at different rotational speeds of spinner wheels, melt flow rates and impingement positions so that the fiberization process was dynamically similar to an industrial mineral wool production process. Images of fiber formation and transport reveal highly complex dynamics of these processes, as fibers mostly occur in form of 3D mutually intertwined structures such as clusters, strands and veils periodically shedding from the melt film. Despite the complexity of flow structures, there is a clear trend of increasing mean fiber length and expansion angle of the coaxial fiber-laden flow as the Weber number and the ratio of melt film velocity to blowing air velocity are increased. The fiberization efficiency (ratio of fiber mass deposited on the collecting mesh to the mass of melt poured) is affected by the impingement position and flow rate of melt as well as the Weber number of melt film. The optimum efficiency was attained at $30^{\circ}\left(10^{\prime} \mathrm{clock}\right.$ ) impingement position and the ratio of melt film to blowing air flow velocity close to unity.

Keywords: spinner, fiber formation, mineral wool, multiphase flow, primary layer, high-speed imaging

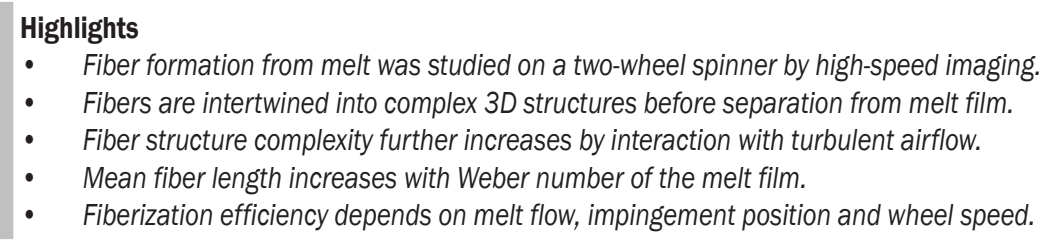

\section{INTRODUCTION}

Turbulent fiber-laden flows are of great importance in manufacturing of fiber-based materials such as mineral wool insulation, paper, composites and nonwoven fabrics [1] and [2]. Fibers for these applications are commonly produced by air-assisted spinning machines (also known as spinners) with two prevailing designs: hollow rotors with perforated walls [3] and [4] and multi wheel spinners where melt cascades between wheels and is fiberized from a free surface [5] and [6]. The former type is used for fiberization of melts with temperatures up to $1000{ }^{\circ} \mathrm{C}$, while the latter type is employed with very high temperature melts (up to $1600{ }^{\circ} \mathrm{C}$ ) in the manufacturing of stone and slag wool and will be the focus of research presented in this paper. The basic operating principle of a multiwheel spinner is the scattering of melt to ligaments from a radial film formed on each wheel, followed by solidification and transport to the fiber deposition area where the primary layer of fibers is formed [5]. Both fiberization and primary layer formation processes are highly complex and not well understood on the micro scale despite the fact that centrifugal fiberization is a mature technology that has been in use for many decades. This is largely due to the fact that mineral fibers are typically very thin (under $10 \mu \mathrm{m}$ ) and initially travelling at velocities in excess of $100 \mathrm{~m} / \mathrm{s}$, effectively preventing the observation of individual fibers even with state-of-the-art high-speed cameras.

To overcome these issues, different approaches have been employed. Simplified theoretical models of fiber formation were proposed by Vad and Morlin [7] and Zhao et al. [8]. Bizjan et al. [9] modelled the fiberization process on a scaled-down, single wheel spinning machine using an isothermal Newtonian liquid. Results of these experiments were successfully reproduced in a numerical simulation by Mencinger et al. [10], while a similar study that also considered solidification of ligament breakup droplets was conducted by Peng et al. [11]. Nevertheless, a full three-dimensional (3D) simulation of the spinner fiberization process has not yet been attempted due to an extreme computational expense when all fiberization-related phenomena are considered. Another possible method for investigation of fiberization and primary layer formation is to employ a low-temperature melt that can be safely handled in the laboratory environment. Several authors investigated the fiberization process as an undesired phenomenon in the dry centrifugal granulation process using a rosin-paraffin melt [12] to [14], but with little regard to fiber formation dynamics. Speaking of the fibrous primary layer formation process, several studies 
have been conducted. Bajcar et al. [15] investigated the dependence of the primary layer texture on aerodynamic characteristics within the collection chamber. Bizjan et al. [16] modelled the bulk density and distribution of deposited sucrose fibers produced by an air-assisted cotton candy machine. A similar study was performed on the collecting drum of an industrial stone wool production line [17]. These studies did not focus on the flight of fibers between the spinner and the collecting mesh. This segment was covered on a more fundamental level by Lin et al. [1] and Capone et al. [18] who modelled transport of short rigid fibers in the axial airflow.

In light of above-presented studies dealing with different stages of fiber formation and transport, the present paper aims to improve the understanding and modelling of realistic fiber formation and transport processes with regard to spinner operating conditions, particularly on small length and time scales. For this reason, a two-wheel spinner resembling industrial spinning machines for production of fibers [5] and powders [19] was designed, and the flow of fibers was investigated experimentally by high-speed imaging.

\section{EXPERIMENTAL SET UP AND METHODOLOGY}

Experimental set-up (Fig. 1) consisted of a spinner with two counter-rotating aluminum wheels, a pivoting cup liquid supply system, blowing and suction air systems, and a high-speed imaging system. The radius of the first spinning wheel was $R_{1}=45 \mathrm{~mm}$, while the second wheel with $R_{2}=52.5 \mathrm{~mm}$ was installed so that the minimum clearance (gap) between wheels was $5 \mathrm{~mm}$ (Fig. 1, front view). Both wheels were $60 \mathrm{~mm}$ long and propelled by a direct-drive electric motor that was powered by a variable frequency drive (VFD), with rotational speed of the wheel varied in the range $40 \mathrm{~Hz}<f<100 \mathrm{~Hz}$. The spinner used in our experiments is a scaled-down version of a fourwheel industrial mineral wool spinning machine (approximately 1:3 scale). Only wheels 1 and 2 were installed for the purpose of the present study as such setup is adequate to investigate fiberization-related phenomena without the unnecessary complexity of the flow visualization setup required for a four-wheel device. Operating parameters in our experiment were selected so that similarity criteria with industrial spinners were satisfied. Three main dimensionless numbers were introduced for this purpose in Eqs. (1) to $(3)$ :

Weber number (ratio of inertial to surface tension forces acting on the melt):

$$
W e=\frac{\rho \cdot \omega^{2} R^{3}}{\gamma} .
$$

Ohnesorge number (ratio of viscous forces relative to inertial and surface tension forces acting on the melt):

$$
O h=\frac{\mu}{\sqrt{\rho R \gamma}} .
$$

Dimensionless velocity ratio:

$$
w=\frac{u}{v_{B}} .
$$

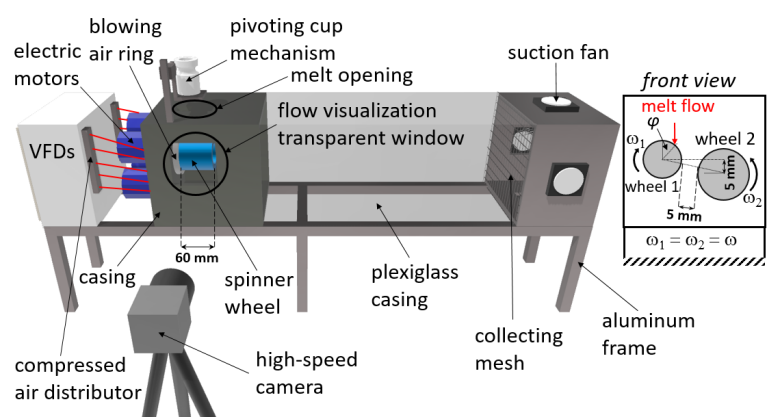

Fig. 1. Experimental set-up for high-speed imaging of melt fiberization

The working medium in our experiments was molten isomalt, a sugar alcohol commonly used in the food industry. The main advantage of using isomalt instead of sucrose is its low susceptibility to caramelization, recrystallization and moisture absorption [20], while its glass transition temperature $\left(T_{\mathrm{g}}=63.6{ }^{\circ} \mathrm{C}[21]\right)$ is sufficiently high to ensure fiber solidification to a glassy state when cooled to ambient temperature. On the other hand, unlike aforementioned rosin-paraffin mixtures, solid isomalt residues can be cleaned with water instead of organic solvents, and the isomalt melt is also safer to handle due to its low volatility and low flammability. The properties of isomalt melt [20] and [21] used in our experiments are provided in Table 1 along with the properties of typical mineral melts [22] and [5]. Also provided in Table 1 is a comparison of $\mathrm{Oh}, \mathrm{We}$ and $w$ dimensionless numbers that confirms a good similarity of model spinner from the present study with industrial spinning machines. Consequently, the findings presented in this paper can be largely applied to the mineral wool manufacturing process.

Once the melt was homogenized at its desired temperature, it was filled into a polytetrafluoroethylene (PTFE) cup with $70 \mathrm{~mm}$ inner diameter (Fig. 2a) preheated to the same temperature to prevent 
Table 1. Comparison of model and industrial spinner parameters, including physical properties of melts and dimensionless similarity criteria for model and industrial spinners

\begin{tabular}{lccccccccc}
\hline Spinner & Material & $T\left[{ }^{\circ} \mathrm{C}\right]$ & $T_{\mathrm{g}}\left[{ }^{\circ} \mathrm{C}\right]$ & $\rho\left[\mathrm{kg} / \mathrm{m}^{3}\right]$ & $\gamma[\mathrm{N} / \mathrm{m}]$ & $\mu[\mathrm{Pa} \cdot \mathrm{s}]$ & $W e[\times 106]$ & $O h$ & $w$ \\
\hline Model & isomalt & 195 & 63.6 & 1418 & 0.070 & 0.21 & 0.19 to 1.16 & 0.09 to 0.10 & 0.44 to 1.1 \\
\hline Indus. & min. melt & 1400 to 1600 & 750 to 900 & 2600 & 0.4 to 0.5 & 0.5 to 1.5 & 0.5 to 10 & 0.05 to 0.15 & 0.5 to 1 \\
\hline
\end{tabular}
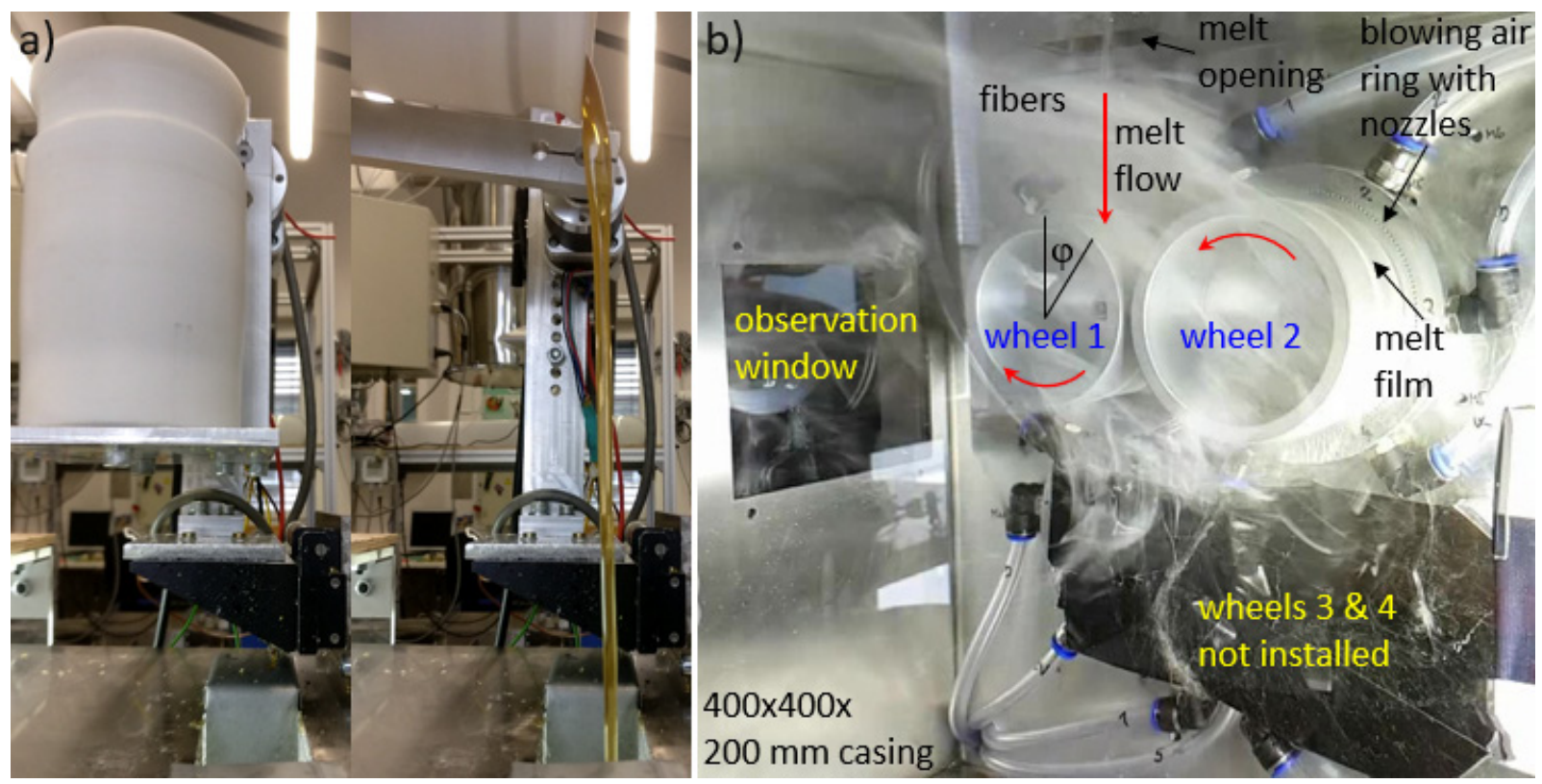

Fig. 2. a) Melt pouring from the pivoting cup, and b) spinner in operation (frontal view)

premature solidification of melt. In all experiments, the mass of the melt batch $\left(m_{\mathrm{M}}\right)$ was approximately $150 \mathrm{~g}$. The cup was then rotated $180^{\circ}$ by a pivoting mechanism powered by a stepper motor, pouring the melt onto the spinner until completely empty. Two different angular velocities were used $(6 \%$ and $9 \%$ ), resulting in a mean volume flow rate of melt $Q=18.3 \mathrm{~mL} / \mathrm{s}$ and $Q=24.8 \mathrm{~mL} / \mathrm{s}$, respectively. The main advantage of using the pivoting cup melt supply is a good repeatability of the pouring process, and a negligible residual mass of melt left in the cup.

The melt flowing from the pivoting cup impacted the first spinner wheel at an impingement angle $\varphi$ (Fig. $2 \mathrm{~b}, \varphi=0^{\circ}$ corresponds to 12 o'clock position on the wheel) and was deflected towards the second wheel. The impingement angle was varied between $15^{\circ}$ and $60^{\circ}$. A radial melt film was formed on both spinning wheels and was continuously disintegrated to melt ligaments that rapidly solidified to fibers. Fibers were transported from the spinner with the aid of coaxial airflow distributed through circular nozzles $(1 \mathrm{~mm}$ diameter, $5 \mathrm{~mm}$ above wheel surface) of two blowing rings (Fig. 2b) connected to a utility compressed air system, and the suction airflow generated by axial fans on the other end of the collection chamber channel. The transparent plexiglass channel was $1 \mathrm{~m}$ long with a $0.5 \mathrm{~m} \times 0.5 \mathrm{~m}$ cross-section. Blowing and suction air flow rates were set to $q_{\mathrm{B}}=10 \mathrm{~L} / \mathrm{s}$ and $q_{\mathrm{S}}=140 \mathrm{~L} / \mathrm{s}$, respectively (ambient air temperature and relative humidity were $23{ }^{\circ} \mathrm{C}$ and $62 \%$, respectively). The resulting ratio $q_{\mathrm{S}} / q_{\mathrm{B}}=14$ was close to ratios used in the mineral wool industry, 10 to 12 , [5]. The velocity of the coaxial blowing air flow above the center of the wheel mantle where melt film was formed was approximately $30 \mathrm{~m} / \mathrm{s}$. As the fiber-laden flow reached the collecting mesh, fibers were deposited, forming a layer of fibrous material also known as the primary layer [16] and [17].

The process of fiberization, fiber flight and primary layer deposition was recorded from the side and top of the spinner casing (fiberization imaging setup shown in Fig. 1) using a high-speed camera (Fastec HiSpec4) with $50 \mathrm{~mm}$ fixed zoom lens and recording at 2500 frames per second. In all cases, a diffuse LED light source was used for illumination of fibrous structures from the opposite side of the focal plane. 


\section{RESULTS AND DISCUSSIONS}

\subsection{Fiberization Process and Fiber Transport}

The process of fiber formation from the melt film was visualized on wheel 2 at three different rotational speeds (Fig. 3) and a $1200 \times 492$ pixels resolution. As seen in Fig. 3 and Table 2, low wheel speeds $(f=40 \mathrm{~Hz}$, $u=13.2 \mathrm{~m} / \mathrm{s}$ ) resulted in fiber trajectories nearly parallel to the wheel surface and a strong recirculation zone behind the wheel, where a significant amount fibers accumulated (note that the front face of both wheels was hollow). A few relatively large droplets generated in the fiberization process (mean radial velocity: $v_{\mathrm{D}, \mathrm{R}}=9.0 \mathrm{~m} / \mathrm{s}$ ) are visible and are mostly transported away by the blowing air flow. In the intermediate range of wheel velocities $(f=70 \mathrm{~Hz}$, $u=23.1 \mathrm{~m} / \mathrm{s}$ ), the recirculation zone can be seen to disappear, and the fibers are transported following a horizontal trajectory downstream of the wheel. However, the number and velocity of melt droplets is increased (Table 2), resulting in droplets penetrating the air flow in the radial plane $\left(v_{\mathrm{D}, \mathrm{R}}=15.5 \mathrm{~m} / \mathrm{s}\right)$.

A further increase of the wheel rotational speed to $100 \mathrm{~Hz}(u=33 \mathrm{~m} / \mathrm{s})$ causes an even larger number and velocity of penetrating melt droplets $\left(v_{\mathrm{D}, \mathrm{R}}=19.1 \mathrm{~m} / \mathrm{s}\right)$. The ratio $v_{\mathrm{D}, \mathrm{R}} / u$ can be seen to decrease with the rotational speed of the wheel, most likely due to increased aerodynamic drag acting on melt droplets. Also decreasing is the mean diameter of droplets $\left(d_{\mathrm{D}}\right)$ that roughly follows the values predicted by the head droplet size model $d_{\mathrm{HD}}=1.95 \cdot R \cdot W e^{-0.45}$ [9], suggesting that most visible droplets are produced by pinching of molten ligament heads. Besides, fibers can also be seen to partly penetrate the coaxial air jet, as indicated by an increased concentration of low velocity fibrous structures below the jet that gradually reenter the blowing air flow. As the circumferential velocity of the wheel increases, radial and tangential velocities of emerging fibers also increase while the axial component does not change significantly (blowing air velocity was kept constant), resulting in a larger spreading angle of the fibrous flow that can be seen as a steeper initial flight path of fibers in Fig. 3. This
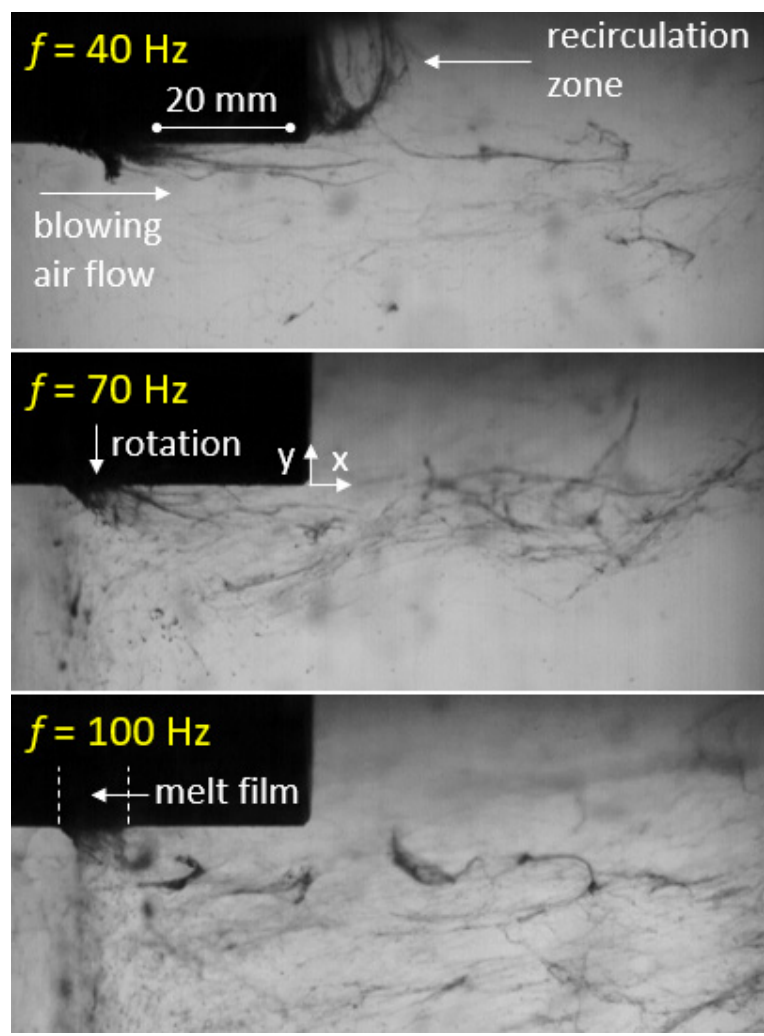

Fig. 3. High-speed images of fiberization process on wheel 2 at different wheel rotational speeds $\left(\varphi=30^{\circ}, Q=24.8 \mathrm{~mL} / \mathrm{s}, O h=0.092\right)$

is also evident from Fig. 4 where vertical profiles of the gray level standard deviation $\sigma_{\mathrm{G}}$ are shown at $x=10 \mathrm{~mm}$ and $x=50 \mathrm{~mm}$ (consider coordinate system in Fig. 3). The value of $\sigma_{\mathrm{G}}$ was normalized to the maximum possible gray level of images (i.e. 255). Besides the shift of the peak $\sigma_{\mathrm{G}}$ position, an increase in wheel rotational speed also results in larger mean values of $\sigma_{\mathrm{G}}$, while the difference between profiles drawn at $x=10 \mathrm{~mm}$ and $x=50 \mathrm{~mm}$ diminishes. Both findings indicate a marked increase in the turbulent intensity of the fiber-laden flow with the rotational speed of the wheel.

A very important parameter for characterization of the fiberization process is the fiber length $L$ (Fig.

Table 2. Dependence of droplet diameters on spinner operating conditions (values calculated for wheel 2)

\begin{tabular}{ccccccccc}
\hline$f[\mathrm{~Hz}]$ & $W e$ & ${ }^{*} v_{\mathrm{D}, \mathrm{R}}[\mathrm{m} / \mathrm{s}]$ & $u[\mathrm{~m} / \mathrm{s}]$ & $w$ & $v_{\mathrm{D}, \mathrm{R}} / u$ & ${ }^{*} d_{\mathrm{D}}[\mathrm{mm}]$ & $d_{\mathrm{HD}}[\mathrm{mm}]$ & ${ }^{*} L_{\mathrm{m}} \pm \sigma_{\mathrm{L}}[\mathrm{mm}]$ \\
\hline 40 & $1.85 \cdot 105$ & 9.00 & 13.2 & 0.44 & 0.682 & 0.41 & 0.436 & $13.1 \pm 5.4$ \\
\hline 70 & $5.67 \cdot 105$ & 15.49 & 23.1 & 0.77 & 0.671 & 0.35 & 0.264 & $14.5 \pm 6.2$ \\
\hline 100 & $1.16 \cdot 106$ & 19.13 & 33.0 & 1.10 & 0.580 & 0.27 & 0.191 & $16.9 \pm 6.5$ \\
\hline
\end{tabular}

* parameters manually measured from images using IrfanView software with on-screen ruler and subpixel interpolation (100 measurements for each set of operating conditions, each measurement accurate to 0.1 pixels / $11 \mu \mathrm{m}$ ). 


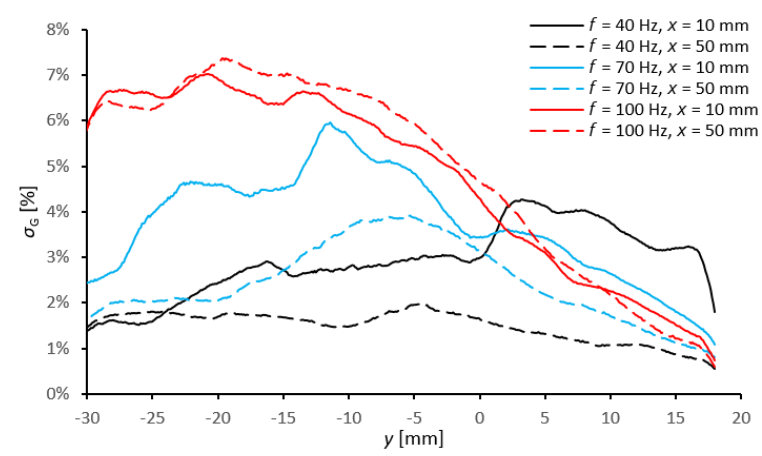

Fig. 4. Standard deviation profiles of the image gray level for different wheel speeds and axial positions

5), and its arithmetic mean $L_{\mathrm{m}}$ (Table 2). Length statistics presented in Table 2 and in Fig. 5 suggest that the fiber length distribution is quite wide, and both the mean and the standard deviation of the length tend to increase with the rotational speed of the wheel. For experiments with the highest Weber number $\left(f=100 \mathrm{~Hz}, W e=1.16 \cdot 10^{6}\right)$, the length of $95 \%$ of fibers was between $10 \mathrm{~mm}$ and $30 \mathrm{~mm}$, and the $L_{\mathrm{m}} / R$ ratio was approximately 0.32 . These figures are consistent with values reported on industrial spinning machines $\left(L_{\mathrm{m}} \approx 10 \mathrm{~mm}, L_{\mathrm{m}} / R=0.3, \ldots, 0.4\right.$; [5]), where

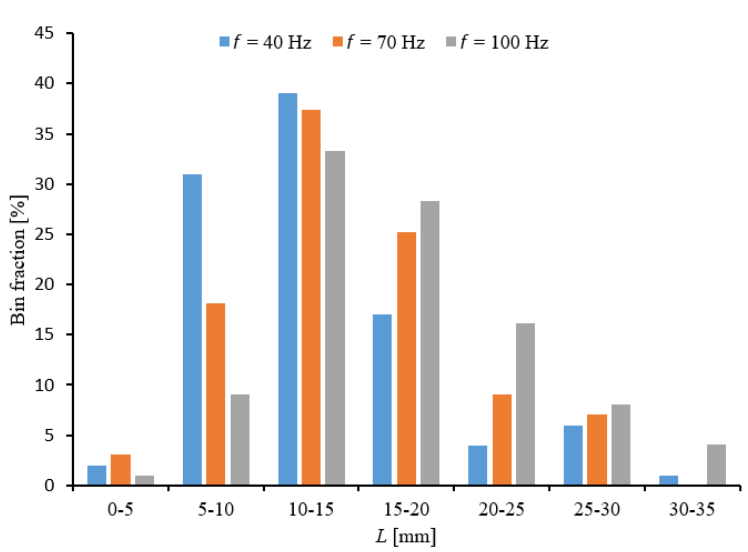

Fig. 5. Histogram of the fiber length based on 100 length measurements at each rotational speed used

fiberization process is hydrodynamically similar to our experiments as it occurs in a similar range of $W e$, $O h$ and $w$ numbers (Table 1). Similarity is considered valid for as long as dimensionless numbers for model and industrial devices are by less than factor 10 apart [5]. Nevertheless, cold experiments performed by Bizjan et al. [23] suggest that much longer fibers are produced in the absence of the blowing air flow, a ratio of $L_{\mathrm{m}} / R \approx 1.1$ was reported on a wheel

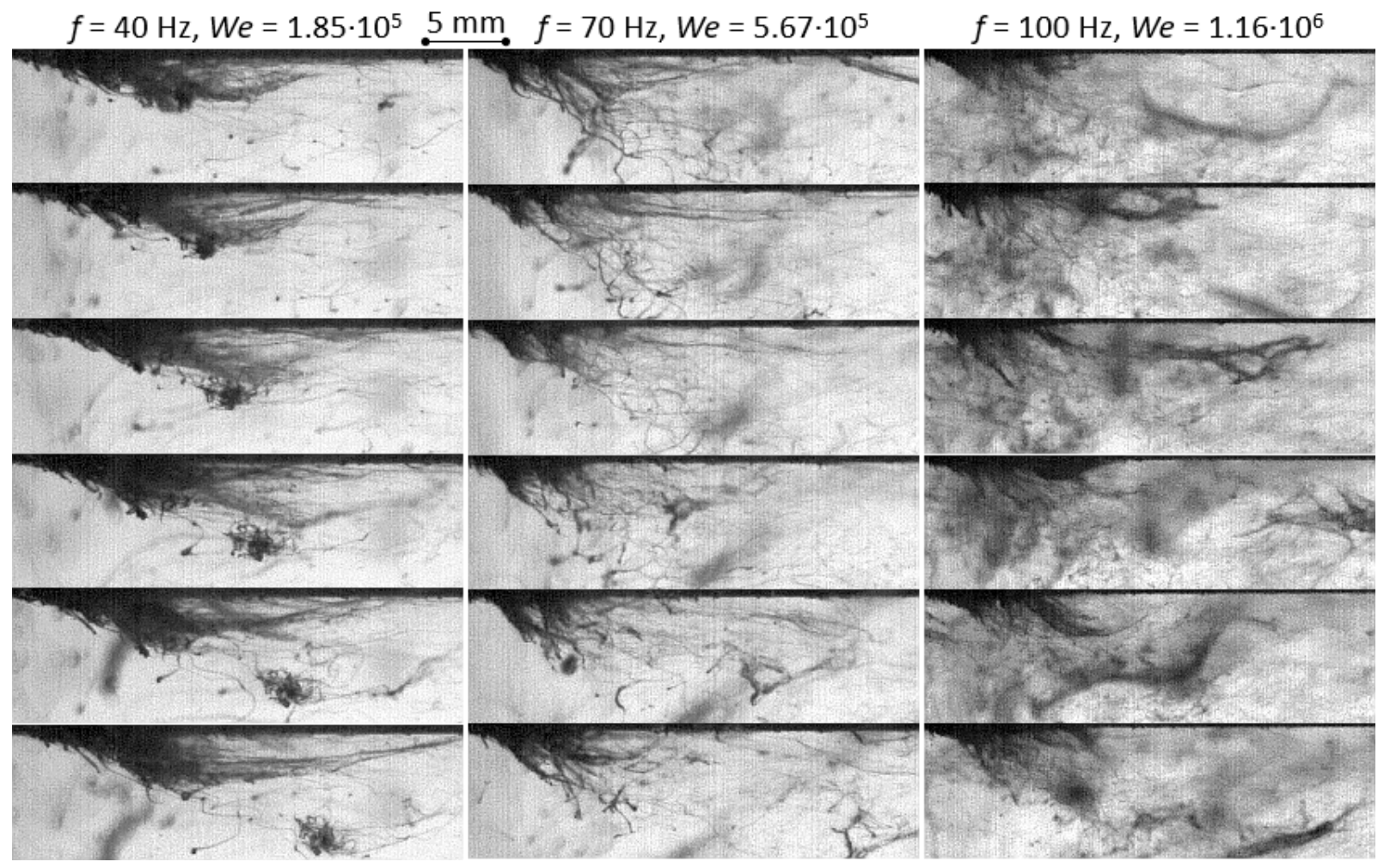

Fig. 6. High-speed images of fiber formation near the melt film $\left(\varphi=30^{\circ}, Q=24.8 \mathrm{~mL} / \mathrm{s}, O h=0.092\right)$, frame separation $0.4 \mathrm{~ms}$ 
with $R=45 \mathrm{~mm}$. This finding is also supported by experience in the mineral wool industry [5], although the exact fiber length is difficult to measure due to the fragility and mutual intertwining of fibers.

A better insight into the microscale dynamics of the fiberization process can be gained by examination of high-speed images in the region adjacent to the melt film (Fig. 6). Regardless of the wheel rotational speed and Weber number, a similar general mechanism can be observed. Liquid ligaments are initially extended from the melt film in a direction perpendicular to the axis of wheel rotation. In this phase, the ligament growth is largely governed by hydrodynamic instabilities such as the Rayleigh-Taylor and KelvinHelmholtz instability [24]. As the ligament head enters the high velocity zone of the coaxial air jet, the ligament is gradually curved towards the axial direction. When the circumferential velocity of the wheel and thus the melt film velocity is much lower than the air flow velocity (e.g. at $f=40 \mathrm{~Hz}$, Fig. 6), most ligaments are immediately deflected towards the axial direction, some reattaching to the wheel surface downstream of the melt film. However, as the ratio between the melt film velocity and the air current velocity approaches unity $(w \approx 1)$, the radial extent of ligaments from the melt film is increased, and the deflection towards the air jet direction is complete at a farther distance from the origin point of ligaments (as already noted, some ligaments can even penetrate the air jet).

Since there is a large number of liquid ligaments attached to the melt film, deflection by the air current inevitably causes ligaments to come in contact, stick to each other and form relatively large clusters upon solidification to fibers. Due to the ongoing ligament formation, such fiber clusters grow continuously while remaining attached to the melt film for extended periods of time. Once a critical size is reached, a part of the cluster is separated under the action of the centrifugal force and the aerodynamic drag force and is transported away from the wheel by the blowing air flow. In our experiments, the most common frequency of fiber cluster shedding was between $150 \%$ and $200 \%$ of the wheel rotational frequency $f$, while the size of both attached and separated clusters significantly decreased with $f$. Apart from the cluster formation, some ligaments that did not immediately intertwine can be seen to extend far downstream of the melt film and solidify to long and thin strands (Fig. 3). Complex fluid-structure interaction phenomena occur as these fiber strands interact with the coaxial air flow and with each other while still attached to the rotating melt film. Eventually, the partly intertwined fiber strands are torn away from the wheel surface, forming veil-like structures of varying sizes and exhibiting a complex 3D motion in the transporting air flow - consider Fig. 7 for flow images recorded from a top-down view immediately downstream of spinning wheels.
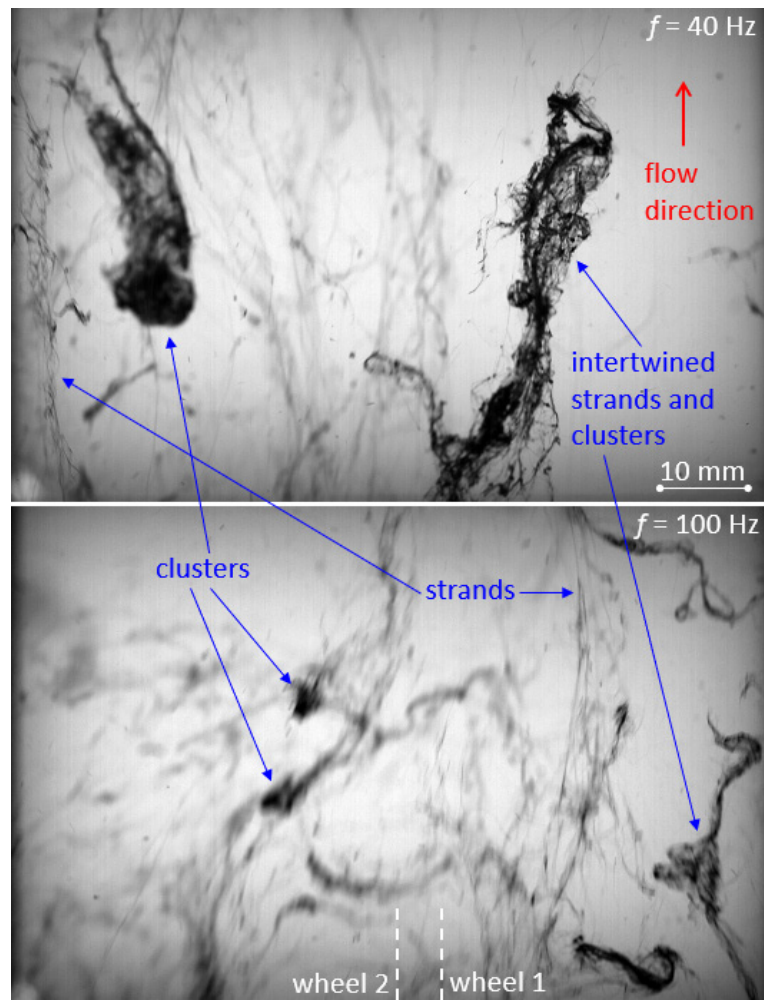

Fig. 7. Typical fibrous structures downstream

of the model spinning machine, top-down view $\left(\varphi=30^{\circ}\right.$,

$Q=24.8 \mathrm{~mL} / \mathrm{s})$; wheel gap position marked by dashed lines

The fact that there is a strong interaction between fibers implies that existing fiberization models assuming a single fiber in the airflow [7], [8] and [25], whilst useful for prediction of fiber diameters, cooling rates and initial trajectories, become inadequate once fibers are mutually intertwined. Particularly the process of fiber tearing from the melt film and subsequent fiber breakage in the blowing air flow is still poorly understood. As noted by Westerlund and Hoikka [25], tensile load calculated by a single fiber model is by an order of magnitude lower than the tensile strength of mineral fibers, while the final fiber length is significantly overestimated. Consequently, it is likely that fiber breaking mechanisms are closely linked to the interaction of fibrous structures with the turbulent air flow that is not considered by any of the known models. 
As already said, the multiphase fiber-laden flow downstream of the spinner is highly complex, a finding confirmed by flow images shown in Fig. 7. Multiple different types of fibrous structures including clusters and strands are visible, often occurring mutually intertwined. Consistent with observations regarding the initial fiberization process (Fig. 6), flow images in Fig. 7 suggest a decreasing trend for the mean size of fibrous structures as the wheel rotational speed is increased. Despite the decreasing size, the bulk density of fiber clusters remains relatively high, as indicated by low light transmittance of these structures (note very dark image areas where the light source is obstructed by clusters).

\subsection{Formation of the Primary Layer of Fibers on the Mesh}

Apart from fiber formation and transport in the airflow, the process of fiber settling on the collecting mesh determines the structure and quality of the primary layer (Fig. 8a) of deposited fibers [5]. The fiber settling process was recorded in the area adjacent to the collecting mesh (Fig. 8 b, image size $880 \times 646$ pixels).

A sample flow image shown in Fig. 8b indicates the presence of complex and interconnected threedimensional fibrous structures near the collecting mesh. As evident from Fig. 9, fibrous structures of very different size and morphology (strands,

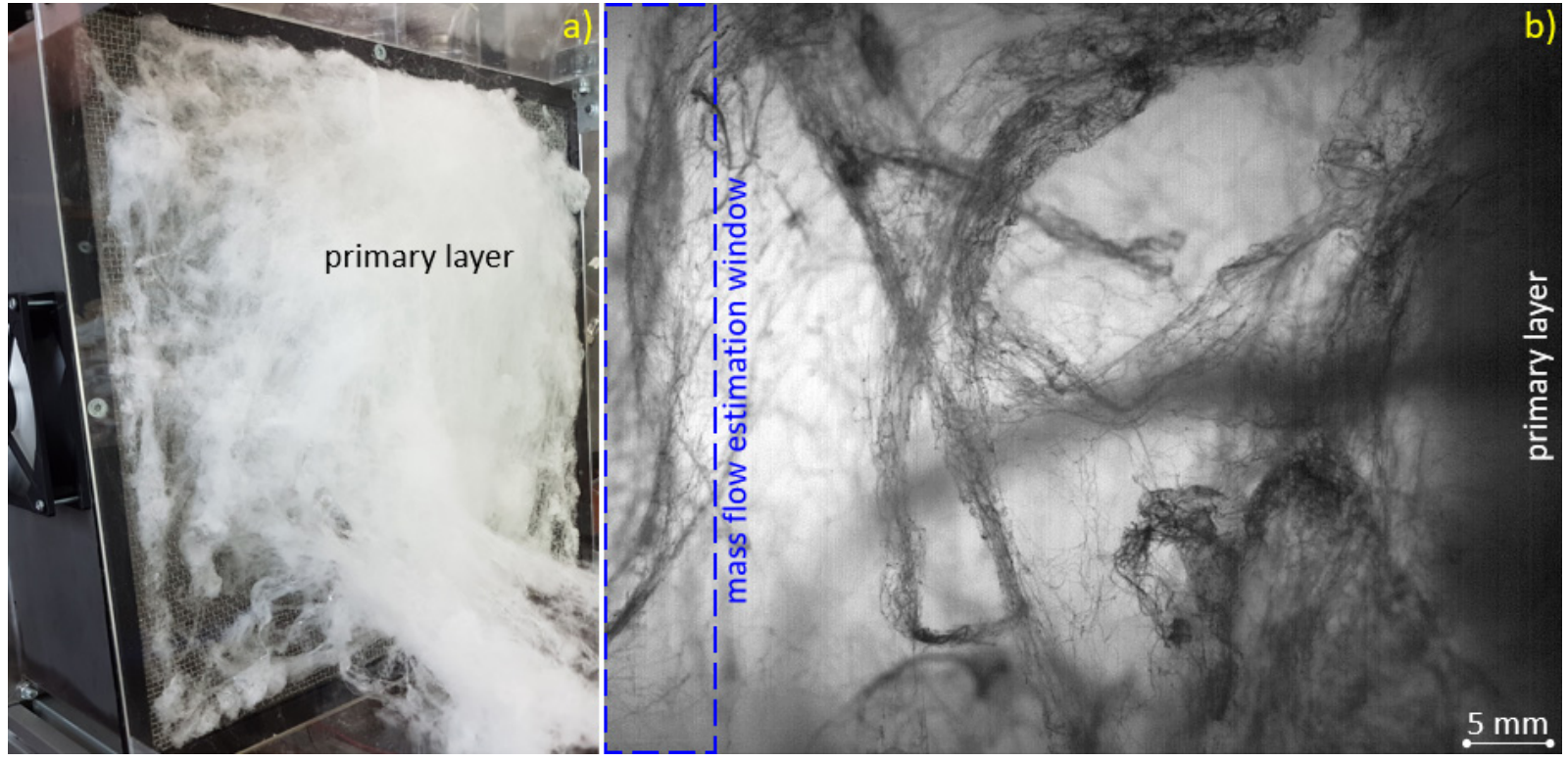

Fig. 8. a) Primary layer of fibers on the collecting mesh; and b) typical fibrous structures in flight near the mesh $\left(f=70 \mathrm{~Hz}, q_{B}=10 \mathrm{~L} / \mathrm{s}, q_{S}=140 \mathrm{~L} / \mathrm{s}\right)$

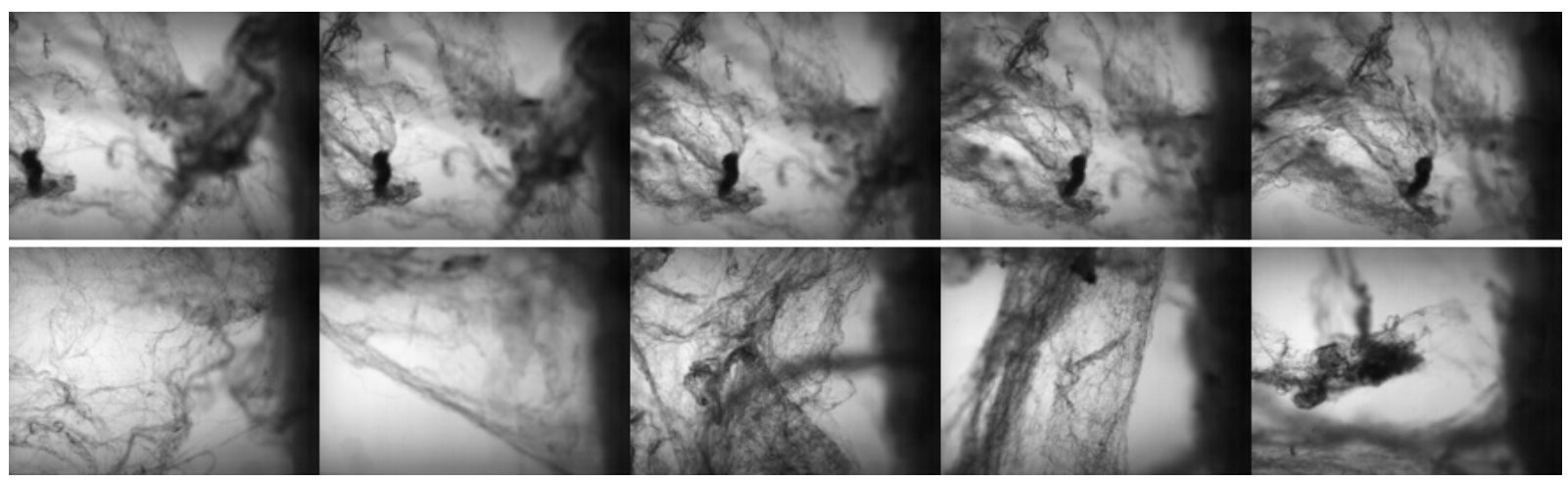

Fig. 9. Fiberization image sequences $\left(f=70 \mathrm{~Hz}, q_{B}=10 \mathrm{~L} / \mathrm{s}, q_{S}=140 \mathrm{~L} / \mathrm{s}\right.$ ); frame separation: $2 \mathrm{~ms}$ (upper row), $100 \mathrm{~ms}$ (lower row); viewing area size: $61.6 \mathrm{~mm} \times 45.2 \mathrm{~mm}$ (all tiles); Note the thickness of the primary layer (right edge of tiles) increasing with time 
veils, clusters) can appear in a single fiberization experiment, including shorter time periods where little or no airborne fibers are observed. Despite the predominant axial velocity component, the motion of fibers is three-dimensional, including rotation and deformation of structures.

High-speed images of the fiber settling process were used to measure the thickness of the primary layer $(h)$ and the relative mass flow rate of fibers $(M) . M$ was calculated as the time series of mean image brightness (leftmost 100 pixels of each image were included, Fig. 8b), and normalized to the value range of $[0, \ldots, 1]$. In Fig. 10, large fluctuations in the value of $M$ can be observed and are consistent with the large fiber structure variability presented in Fig. 9. However, the estimated cumulative mass of deposited fibers $I_{\mathrm{m}}$ calculated by temporal integration of $M$ increases in a fairly linear manner with time despite the mass flow rate fluctuations, suggesting a quasisteady fiber deposition process. A similar linear trend can be observed for the growth of the primary layer thickness $h$. Visible oscillations of $h$ (rapid increases followed by periods of fairly constant $h$ ) are likely related to spatially random thickening of the primary layer, as well as redistribution and fluttering of large fiber structures on the mesh, all leading to alternating positive and negative errors in measurement of $h$. Oscillations also indicate the possibility of unsteady rate of layer compression, although further research is needed in this regard.

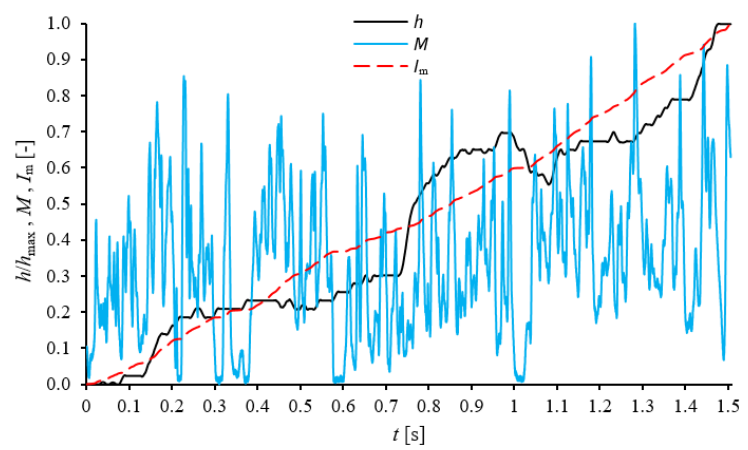

Fig. 10. Relative mass flow rate, relative deposited mass of fibers and primary layer thickness ( $f=70 \mathrm{~Hz}$, $q_{B}=10 \mathrm{~L} / \mathrm{s}, q_{B}=140 \mathrm{~L} / \mathrm{s}, h_{\max }=15.1 \mathrm{~mm}$ )

The fact that the time-averaged growth rate of the thickness $h$ was fairly constant (approximately $9.2 \mathrm{~mm} / \mathrm{s}$ ) indicates that compression of fibrous structures mostly occurred when these structures settled on the collecting mesh, while subsequent additional compression due to deposition of new fibers and resulting increased pressure difference across the mesh was negligible. This can be explained by a low suction pressure in our experiments (approximately $15 \mathrm{~Pa}$ when mesh was covered by fibers). On the other hand, high-capacity industrial collecting chambers where the mesh is moving often operate with suction pressures in excess of $500 \mathrm{~Pa}$, resulting in a higher degree of primary layer compression.

After each fiberization experiment, fibers were removed from the mesh and weighed. Collected fiber mass $m_{\mathrm{F}}$ was used to calculate the fiberization efficiency $\eta_{0}=m_{\mathrm{F}} / m_{\mathrm{M}}$ (Fig. 11) and its relative value $\eta$ calculated by normalization to the peak efficiency of a given curve. Curves presented in Fig. 11 suggest that fiberization efficiency initially increases with the rotational speed of spinner wheels and melt impingement angle. After passing an optimal setting $\left(\varphi \approx 30^{\circ}, f=65 \mathrm{~Hz}\right.$ to $\left.80 \mathrm{~Hz}\right), \eta$ begins to decrease.

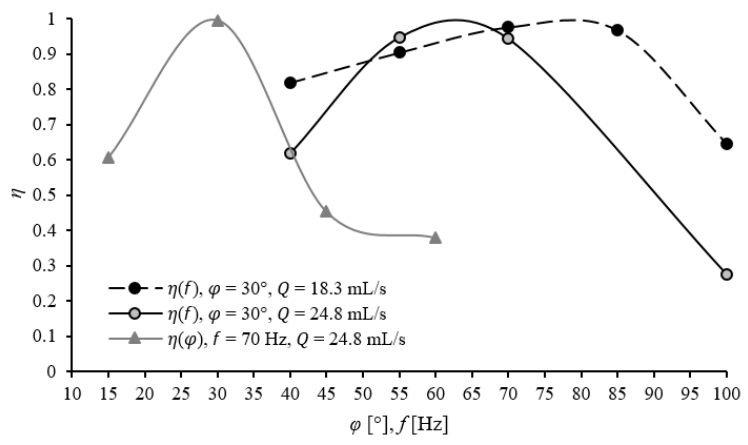

Fig. 11. Diagrams of relative fiberization efficiency as a function of wheel rotational speed, melt impingement position and flow rate time

At $\varphi=30^{\circ}$, the $\eta(f)$ efficiency curve of the lower flow rate used $(Q=18.3 \mathrm{~mL} / \mathrm{s})$ exhibits a narrower range of efficiency than the curve for $Q=24.8 \mathrm{~mL} / \mathrm{s}$, and is less sensitive to non-optimal wheel speed settings. This suggests that the higher flow rate used in our experiments is near the maximum flow rate of melt that can be fiberized in the present spinner arrangement, and deviations from optimal settings of $f$ and $\varphi$ result in a significant mass fraction of melt remaining unfiberized. When the melt impingement position is low $\left(\varphi \geq 45^{\circ}\right)$, a large part of melt flows through the spinner wheel gap without adhering to the melt film [5]. Since wheels 3 and 4 were not installed in this study, this melt could not be fiberized once passing through the gap and was therefore lost. A different melt loss mechanism occurs when melt flow rates are used with high impingement positions, though. In this case, a significant proportion of melt 
is lost immediately after the impingement due to the splashing [5]. The splashing phenomenon can be explained by the occurrence of a hydraulic jump [26] when a stream of liquid or melt impinges a highspeed moving surface. The study by Keshavarz et al. [26] was conducted by liquid impact on a horizontal moving surface, while in our case the melt impacts a rotating surface with a relatively low curvature radius and is partly deflected towards the second spinning wheel. We suspect that the detachment of the melt jet with superimposed hydraulic jump structures may further intensify the splashing, and should be researched in future studies.

\section{CONCLUSIONS}

In this work, the process of melt fiberization and primary layer formation was studied on a two-wheel spinner. The effect of operating parameter variation including rotational speed of spinner wheels, melt impingement position and flow rate was investigated by high-speed imaging of the fibrous flow at different locations in the collection chamber channel.

The ratio of melt film velocity to blowing air velocity, as well as the melt film Weber number significantly affect fiber trajectories and structure, mean fiber length and fiberization efficiency, with optimum fiberization quality and efficiency near $w=1$ and $\log (W e) \approx 6$. The fiberization efficiency is also governed by the impingement position of the melt on the spinner and is highest when $\varphi \approx 30^{\circ}$. The mean fiber length and its standard deviation both gradually increase with $w$ and $W e$, but fibers produced are relatively short when $w \approx 1\left(L_{\mathrm{M}} / R \approx 0.3\right)$.

The fiber formation and breakage process cannot be adequately described by single-fiber models due to the fact that molten ligaments and solidified fibers mutually intertwine in complex 3D structures such as clusters, strands and veils. These structures interact with the turbulent blowing air flow and are torn away from the melt film with a mean frequency higher than the rotational frequency of spinner wheels.

The complexity and randomness of fibrous flow structures increases from the spinner towards the collecting mesh where the primary layer is formed. Nevertheless, the primary layer formation is quasisteady when observed on a sufficiently long timescale, and there is little compression of existing layer when new fibers are accumulated.

Further research should investigate the effect of the blowing air velocity on fiberization. Also relevant for more in-depth research is the process of primary layer formation, particularly with regard to the influence of suction flow velocity and pressure on the structure and density of deposited fibrous layer.

\section{ACKNOWLEDGEMENTS}

The authors acknowledge the financial support from the Slovenian Research Agency - ARRS (research core funding No. P2-0401, and grant Z7-8271).

\section{NOMENCLATURES}

$G$ image gray level, [-]

$h$ primary layer thickness, [m]

$h_{\max }$ maximum primary layer thickness, [m]

$I_{\mathrm{m}}$ relative cumulative mass of deposited fibers, [-]

$L$ fiber length, [m]

$L_{\mathrm{m}}$ mean fiber length, [mm]

$m_{\mathrm{F}}$ mass of fibers collected on the mesh, [kg]

$m_{\mathrm{M}}$ mass of poured melt batch, $[\mathrm{kg}]$

$M$ relative mass flow of fibers, [-]

Oh Ohnesorge number, [-]

$Q$ volume flow rate of melt, $\left[\mathrm{m}^{3} / \mathrm{s}\right]$

$q_{\mathrm{B}}$ volume flow rate of blowing air flow, $\left[\mathrm{m}^{3} / \mathrm{s}\right]$

$q_{\mathrm{S}}$ volume flow rate of suction air flow, $\left[\mathrm{m}^{3} / \mathrm{s}\right]$

$R$ spinner wheel radius, [m]

$t$ time since the start of melt pouring, [s]

$T$ melt pouring temperature, $\left[{ }^{\circ} \mathrm{C}\right]$

$T_{\mathrm{g}}$ melt glass transition temperature, $\left[{ }^{\circ} \mathrm{C}\right]$

$u$ wheel circumferential velocity, $[\mathrm{m} / \mathrm{s}]$

$v$ melt velocity, $[\mathrm{m} / \mathrm{s}]$

$v_{\mathrm{B}}$ blowing air velocity, $[\mathrm{m} / \mathrm{s}]$

$w$ ratio of wheel $/$ melt film circumferential velocity to blowing air velocity, [-]

$W$ acquisition window width, [m]

We Weber number, [-]

$\gamma$ melt surface tension, $[\mathrm{N} / \mathrm{m}]$

$\eta \quad$ relative fiberization efficiency, [-]

$\mu$ melt viscosity, $[\mathrm{Pa} \cdot \mathrm{s}]$

$\rho$ melt density, $\left[\mathrm{kg} / \mathrm{m}^{3}\right]$

$\varphi \quad$ liquid impingement position, $\left[{ }^{\circ}\right]$

$\sigma_{\mathrm{G}}$ normalized standard deviation of image gray level, [\%]

$\sigma_{\mathrm{L}}$ normalized standard deviation of fiber length, [\%]

$\omega$ angular velocity of the wheel, $[\mathrm{rad} / \mathrm{s}]$

\section{REFERENCES}

[1] Lin, J.Z., Liang, X.Y., Zhang, S.L. (2012). Numerical simulation of fiber orientation distribution in round turbulent jet of fiber suspension. Chemical Engineering Research and Design, vol. 90, no. 6, p. 766-775, Dol:10.1016/j.cherd.2011.09.016. 
[2] Zhang, Z.Q., Zhang, Z.Y., Yang, A.M., Xing, H.W. (2015). Preparation and properties of slag wool board using modified polyvinyl alcohol as binder. Materials and Manufacturing Processes, vol. 31, no. 2, p. 168-172, D0l:10.1080/1042691 4.2015.1019128.

[3] Marheineke, N., Liljo, J., Mohring, J., Schnebele, J., Wegener, R. (2012). Multiphysics and multimethods problem of rotational glass fiber melt-spinning. International Journal of Numerical Analysis and Modeling, Series B, vol. 3, no. 3, p. 330-344.

[4] Qin, Y., Lv, X., Bai, C., Chen, P., Qiu, G., Zhang, J. (2014). Mechanism of dry molten slag granulation using a rotating multi-nozzle cup atomizer. Steel Research International, vol. 85, no. 1, p. 44-52, D0l:10.1002 /srin.201300007.

[5] Širok, B., Blagojevič, B., Bullen, P. (2008). Mineral Wool: Production and Properties. Woodhead, Cambridge.

[6] Singha, K. (2012). A short review on basalt fiber. International Journal of Textile Science, vol. 1, no. 4, p. 19-28, DOI:10.5923/j.textile.20120104.02.

[7] Vad, J., Morlin, B. (2007). Fluid mechanical model for formation of mineral wool fibers applied in polymer composites. Materials Science Forum, vol. 537-538, p. 269276, D0I:10.4028/www.scientific.net/MSF.537-538.269.

[8] Zhao, D., Zhang, Z., Liu, L., Wang, X. (2015). A novel kinematic model for molten slag fiberization: Prediction of slag fiber properties. Metallurgical and Materials Transactions B, vol. 46, p. 993-1001, D0l:10.1007/s11663-014-0259-9.

[9] Bizjan, B., Širok, B., Hočevar, M., Orbanić, A. (2014). Ligamenttype liquid disintegration on a spinning wheel. Chemical Engineering Science, vol. 116, p. 172-782, D0I:10.1016/j. ces.2014.08.031.

[10] Mencinger, J., Bizjan, B., Širok, B. (2015). Numerical simulation of ligament-growth on a spinning wheel. International Journal of Multiphase Flow, vol. 77, p. 90-103, D0l:10.1016/j. ijmultiphaseflow.2015.0 8.002.

[11] Peng, H., Hu, Z., Shan, X., Ling, X., Liu, L. (2019). Study on the solidification characteristics of molten slag droplets cooled by mixed cooling medium. Applied Thermal Engineering, vol. 149, p. 939-949, D0I:10.1016/j.applthermaleng.2018.12.115.

[12] Zhu, X., Zhang, H., Tan, Y., Wang, H., Liao, O. (2015). Analogue experimental study on centrifugal-air blast granulation for molten slag. Applied Thermal Engineering, vol. 88, p. 157164, D0I:10.1016/j.applthermaleng.2014.11.077.

[13] Dhirhi, R., Prasad, K., Shukla, K.A., Sarkar, S., Renganathan, T., Pushpavanam, S., Kaza, M. (2016). Experimental study of rotating dry slag granulation unit: Operating regimes, particle size analysis and scale up. Applied Thermal Engineering, vol. 107, p. 898-906, D0l:10.1016/j.applthermaleng.2016.07.049.

[14] Peng, H., Shan, X., Ling, X., Wang, D., Li, J. (2018). Analogue experimental investigation on ligament granulation of molten slag in various rotary disk configurations for waste energy recovery. Results in Physics, vol. 11, p. 385-393, DOI:10.1016/j.rinp.2018.09.037.
[15] Bajcar, T., Blagojevič, B., Širok, B., Dular, M. (2007). Influence of flow properties on a structure of a mineral wool primary layer. Experimental Thermal and Fluid Science, vol. 32, p. 440-449, D0I:10.1016/j.expthermflusci.2007.05.007.

[16] Bizjan, B., Peternelj, M., Širok, B. (2016). Mineral wool primary layer formation in collecting chamber. Strojniški vestnik Journal of Mechanical Engineering, vol. 62, no. 3, p. 179-186, DOI:10.5545/sv-jme.2015.2995.

[17] Peternelj, M., Bizjan, B., Širok, B. (2017). The influence of airflow characteristics and accumulation grid velocity on the formation of a stone wool primary layer. Strojniški vestnik Journal of Mechanical Engineering, vol. 63, no. 6, p. 405-414, DOI:10.5545/sv-jme.2017.4503.

[18] Capone, A., Romano, G.P., Soldati, A. (2015). Experimental investigation on interactions among fluid and rod-like particles in a turbulent pipe jet by means of particle image velocimetry. Experiments in Fluids, vol. 56, art ID. 1, D0I:10.1007/s00348014-1876-4.

[19] Singer, A.R.E., Roche, A.D., Day, L. (1980). Atomization of liquid metals using twin roller technique. Powder Metallurgy, vol. 23, no. 2, p. 81-85, D0I:10.1179/pom.1980.23.2.81.

[20] Hartel, R.W., Ergun, R., Vogel, S. (2011). Phase/state transitions of confectionery sweeteners: Thermodynamic and kinetic aspects. Comprehensive Reviews in Food Science and Food Safety, vol. 10, p. 17-32, Dol:10.1111/j.1541 4337.2010.00136.x.

[21] Raudonus, J., Bernard, J., Janßen, H., Kowalczyk, J., Carle, R. (2000). Effect of oligomeric or polymeric additives on glass transition, viscosity and crystallization of amorphous isomalt. Food Research International, vol. 33, no. 1, p. 41-51, DOI:10.1016/S0963-9969(00)00022-3.

[22] Allibert, M., Gaye, H., Geiseler, J., Janke, D., Keene, B.J., Kirner, D., Kowalski, M., Lehmann, J., Mills, K.C., Neuschütz, D., Parra, R., Saint-Jours, C., Spencer, P.J., Susa, M., Tmar, M., Woermann, E. (1995). Slag Atlas. Verlag Stahleisen, Düsseldorf.

[23] Bizjan, B., Širok, B., Hočevar, M., Orbanić, A. (2014). Liquid ligament formation dynamics on a spinning wheel. Chemical Engineering Science, vol. 119, p. 187-198, D0l:10.1016/j. ces.2014.08.031.

[24] Liu, J., Yu, Q., Guo, Q. (2012). Experimental investigation of liquid disintegration by rotary cups. Chemical Engineering Science, vol. 73, p. 44-50, Dol:10.1016/j.ces.2012.01.010.

[25] Westerlund, T., Hoikka, T. (1989). On the modeling of mineral fiber formation. Computers \& Chemical Engineering, vol. 13, no. 10, p. 1153-1163, Dol:10.1016/0098-1354(89)87018-8.

[26] Keshavarz, B., Green, S.I., Davy, M.H., Eadie, D.T. (2011). Newtonian liquid jet impaction on a high-speed moving surface. International Journal of Heat and Fluid Flow, vol. 32, no. 6, p. 1216-1225, D0l:10.1016/j.ijheatfluidflow.2011.08.001. 